\title{
Aquaporin 8 mRNA expression after intestinal resection in
}

\section{rat}

\author{
Yu Koyama ${ }^{*}$, Hitoshi Kameyama ${ }^{1}$, Jun Sakata ${ }^{1}$, Takashi Kobayashi ${ }^{1}$, Masahiro Minagawa ${ }^{1}$, \\ Shin-ichi Kosugi ${ }^{1}$, Tadashi Yamamoto ${ }^{2}$, Kohei Akazawa ${ }^{3}$, Toshifumi Wakai ${ }^{1}$ \\ ${ }^{1}$ Division of Digestive \& General Surgery, Niigata University Graduate School of Medical \& Dental Sciences, Niigata, Japan \\ ${ }^{2}$ Department of Renal Pathology, Institute of Nephrology, Niigata University Graduate School of Medical \& Dental Sciences, Niigata, \\ Japan \\ ${ }^{3}$ Department of Medical Informatics, Niigata University Graduate School of Medical \& Dental Sciences, Niigata, Japan \\ Email: *yukmy@med.niigata-u.ac.jp
}

Received 2 January 2014; revised 3 February 2014; accepted 9 February 2014

Copyright (C) 2014 Yu Koyama et al. This is an open access article distributed under the Creative Commons Attribution License, which permits unrestricted use, distribution, and reproduction in any medium, provided the original work is properly cited. In accordance of the Creative Commons Attribution License all Copyrights @ 2014 are reserved for SCIRP and the owner of the intellectual property Yu Koyama et al. All Copyright (C 2014 are guarded by law and by SCIRP as a guardian.

\section{ABSTRACT}

Backgrounds: Aquaporins (AQPs), the mammalian water channels, have been localized in various organs, including the gastrointestinal (GI) tract. We examined AQPs expression in rat models of massive intestineal resection to determine the functions of AQPs in the GI tract. Methods: Female Sprague-Dawley rats $(n=15)$ underwent $90 \%$ resection of the small intestine, and Female Wistar-Kyoto rats $(n=10)$, received subtotal colectomy, and were sacrificed following the operations. RNase protection assay and quantitative reverse transcription-polymerase chain reaction (RTPCR) were performed to measure the AQPs mRNA expression in the GI tract. Immunohistochemistry was performed to confirm AQP8 protein expression. Results: AQP8 mRNA expression (mean \pm standard error), was enhanced in the jejunum of the short bowel rats at days 7 and $14(37.6 \% \pm 1.4 \%$ and $18.5 \% \pm$ $2.4 \%$, respectively, $\mathrm{p}<0.01)$. Enhancement of AQP8 mRNA was also observed in the remnant rectum of the subtotal colectomized rats at both days 21 and 42 (116.1\% $\pm 4.5 \%$ and $143.3 \% \pm 7.4 \%$, respectively, $p<$ 0.01). Immunohistochemistry demonstrated enhanced AQP8 expression in the remnant rectum of the subtotal colectomized rats. No intensive change was observed with other AQPs in both models. Conclusions: Our results suggest a compensatory role of AQP8 in the maintenance of intestinal fluid balance.

\section{KEYWORDS}

Aquaporin 8; Short Bowel; Subtotal Colectomy; RNase Protection Assay; QuantitativeRT-PCR

\footnotetext{
"Corresponding author.
}

\section{INTRODUCTION}

Water-selective channels (aquaporins: AQPs) have been identified as molecules located mainly on the plasma membrane of various cell types and increase water permeability [1]. The mammalian water channel, AQP1, was first identified as a homologous protein to MIP (major intrinsic protein of bovine lens) in erythrocytes [1,2] and was also shown to be present in the proximal tubule and the thin descending limb of Henle in the kidney. By means of the homology cloning technique, AQP2 through 10 have been cloned from various organs: AQP2 [3] and AQP3 [4] from the kidney, AQP4 from the brain [5], AQP5 from the salivary gland [6], AQP6 from the kidney [7], AQP7 from the testis [8], AQP8 from the pancreas and liver [9], AQP9 from the liver [10] and AQP10 from the small intestine [11].

Recent studies have demonstrated expression of several types of AQPs in the gastrointestinal (GI) tract, suggesting their participation in water absorption or secretion $[1,4,5,9]$. We have demonstrated both mRNA and protein expressions of AQP1, AQP3, AQP4 and AQP8 in the rat GI tract $[12,13]$. However, the functions of AQPs in the GI tract have not yet been well elucidated.

In the present study, we examined the mRNA expression of AQPs in rat models of massive resection of the small intestine or subtotal colectomy in order to investigate the possible functions of AQPs in the GI tract during postoperative adaptation to intestinal resection.

\section{METHODS}

\subsection{Experimental Animals}

All experiments and surgical procedures conformed to 
the guidelines for the proper care and use of laboratory animals provided by the Public Health Service, National Institutes of Health. For the short bowel model, female Sprague-Dawley (SD) rats weighing approximately 270 g were purchased from Charles River Japan (Yokohama, Japan). Female Wistar-Kyoto (WKY) rats weighing approximately $120 \mathrm{~g}$ were also obtained for the subtotal colectomy model. Throughout the experimental period except for $12 \mathrm{~h}$ prior to the surgical procedures, the animals were given free access to standard lab rat chow and tap water. Animal conditions and weights were recorded daily.

\subsection{Short Bowel Model}

The rats were divided into three groups. The Group SB ( $n=15$ ) underwent an operation to resect about $90 \%$ of the small intestine with primary anastomosis. Group Sham-SD $(\mathrm{n}=15)$ underwent a sham operation, and Group N ( $n=4)$ acted as a normal control without any surgical procedure. Animals were denied food for 12 hours and were anesthetized by inhalation of diethylether and intraperitoneal injection of pentobarbital. In Group $\mathrm{SB}, \sim 90 \%$ of the small intestine was resected from $5 \mathrm{~cm}$ distal to the ligament of Treitz to a point $5 \mathrm{~cm}$ proximal to the ileocecal valve, and underwent jejunoileal anastomosis. In Group Sham-SD, the jejunum was divided at 5 cm distal to the ligament of Treitz, and underwent jejunojejunal anastomosis without removal of the small intestine. All bowel anastomoses were completed with five interrupted 5 - 0 silk sutures. The abdominal wound was closed in two running layers, using 2 - 0 silk.

Two rats of Group SB and 2 of Group Sham-SD died from anesthesia failure, and 2 rats of Group SB and 1 of Group Sham-SD died from anastomosis failure.

The animals of both Group SB and Group Sham-SD were sacrificed at day 7 ( $n=4$ and $n=4$, respectively), day 14 ( $n=3$ and $n=4$, respectively), and day $28(n=4$ and $n=4$, respectively) after the operation. In Group N, the animals were sacrificed at day 28. Total RNAs were isolated from the lower portions of the jejunum, ileum, and proximal and distal colons using a modified acid guanidiniumthiocyanate phenol-chloroform extraction method by using TRIzol (GIBCO BRL, Life Technologies, Rockville, MD, USA) as described previously [12].

\subsection{Subtotal Colectomy Model}

Female Wistar-Kyoto rats weighing about $120 \mathrm{~g}$ were divided into two groups. Group TC $(\mathrm{n}=10)$ underwent subtotal colectomy with primary anastomosis, and Group S-WKY $(n=8)$ underwent a sham operation. Animals were denied food for 12 hours and were anesthetized as described above. In Group TC, a short segment of the ileum and all the large intestine was resected from $2 \mathrm{~cm}$ proximal to the ileocecal valve to $2 \mathrm{~cm}$ proximal to the anal verge, and underwent ileorectal anastomosis. In Group $\mathrm{S}-\mathrm{WKY}$, the ileum was divided at $2 \mathrm{~cm}$ proximal to the ileocecal valve, and underwent ileoileal anastomosis without removal of the small or the large intestine. All bowel anastomoses were completed as described above.

One rat of Group TC and one in Group S-WKY died from anesthesia failure; however, no rat died from anastomosis failure.

The animals of both Group TC and Group S-WKY were sacrificed at postoperative day 21 ( $n=4$ and $n=4$, respectively) and day 42 ( $n=5$ and $n=3$, respectively). Total RNAs were isolated from the jejunum, the middle portion of the small intestine, the ileum, and the remnant rectum as described above.

\subsection{PCR Cloning of Rat Aquaporins}

Rat AQP1 (356 bp; +226 + 581), rat AQP3 (377 bp; +256 +632) and rat AQP4 (330 bp; +302 +631) cDNA fragments were obtained from the rat colon (AQP1 and AQP3) or ileum (AQP4) by the PCR-based cloning method using the nested, degenerate oligonucleotide primers for the AQP family as reported previously [12]. The PCR products were subcloned into pGEM Tvectors (Promega Japan Inc., Tokyo, Japan) and their sequences were verified using an automated DNA sequencer (Perkin Elmer, Foster City, CA, USA). A partial fragment of rat glyceraldehyde-3-phosphate dehydrogenase (GAPDH) cDNA of 123 bp was inserted in pGEM 3Z (Promega). Rat AQP8 cDNA (315 bp; +701 +1015) was subcloned into pGEM 11Z (Promega) using a method reported previously $[9,12]$.

The plasmids inserted with the rat AQP genes were linearized with appropriate restriction enzymes and used as templates for the in vitro transcription of $\left[{ }^{32} \mathrm{P}\right]$-labeled antisense cRNA probes.

\subsection{Ribonuclease Protection Assay}

Ribonuclease (RNase) protection assay was carried out as described previously [9,12]. In brief, $10 \mathrm{~g}$ of each total RNA sample was hybridized with the AQP and GAPDH probes containing a specific radioactivity of $1 \times 10^{5} \mathrm{cpm}$ each in $10 \mathrm{ml}$ of hybridization buffer (80\% formamide, $40 \mathrm{mM}$ PIPES, $0.4 \mathrm{M} \mathrm{NaCl}, 1 \mathrm{mM}$ EDTA) overnight at $48^{\circ} \mathrm{C}$. Unhybridized probes were then digested with RNase $\mathrm{A}$ and RNase $\mathrm{T} 1$ at $30^{\circ} \mathrm{C}$ for 1 hour, and the RNases were digested with proteinase $\mathrm{K}$ at $37^{\circ} \mathrm{C}$ for 30 minutes. After phenol/chloroform extraction, the hybridized probes were precipitated with ethanol, denatured at $85^{\circ} \mathrm{C}$, and electrophoresed on $6 \%$ polyacrylamide gels. The dried gels were exposed to X-ray films (Fuji Photo Film Co., Kanagawa, Japan) for 3 days. After developing, the autoradiography films were optically scanned by HP 
ScanJet 3C (Hewlett- Packard Co., Greeley, CO, USA) and the density of each band was analyzed by a computerized densitometry using Power Macintosh 9500/132 (Apple Computer, Cupertino, CA, USA) employing NIH Image software version 1.59 (NIH Division of Computer Research and Technology, Bethesda, MD, USA). The data are represented as mean \pm standard error (SE) of ratios $(\mathrm{AQP} / \mathrm{GAPDH}$ mRNA densitometric unit) $\times 100 \%$, as reported previously [12].

\subsection{Quantitative RT-PCR}

Because significant changes were observed only in AQP8 mRNA expression in both experimental models by the RNase protection assay, further examination of AQP8 mRNA expression of both experimental models by quantitative RT-PCR assay was performed.

Total RNA (1 g) of each sample was reverse-transcribed at $42^{\circ} \mathrm{C}$ for $1 \mathrm{~h}$ by using an oligo (dT) primer and Superscript II reverse transcriptase (GIBCO BRL) in a volume of $20 \mu \mathrm{l}$. Each reverse-transcript was used as a template for multiplex quantitative PCR to measure each AQP mRNA and GAPDH expression in a single well by means of an ABI PRISM 7700 Sequence Detection Instrument (PE Biosystems Japan, Chiba, Japan).

The AQP8-specific primers were

5'-GGCAGGTGGTGGGATCTCT-3' and

5'-GCCTAATGAGCAGTCCCACAA-3', and the fluo rogenic probe was

5'-TGGATCTACTGGCTGGGCCCACTC-3'. A

GAPDH TaqMan Rodent GAPDH Control Reagent VIC $^{\mathrm{TM}}$ Probe (PE Biosystems Japan) was used for amplifying rat GAPDH for use as an internal control.

Amplification reactions $(50 \mu \mathrm{l})$ contained a reversetranscript $(1 \mu \mathrm{l}), 1 \times$ TaqMan Universal PCR Master Mix (PE Biosystems), $900 \mathrm{nM}$ of each AQP8 primer, $250 \mathrm{nM}$ of AQP8 fluorogenic probe, $100 \mathrm{nM}$ of rodent GAPDH primers, and $250 \mathrm{nM}$ of rodent GAPDH fluorogenic probe. All quantitative 2 step-PCR reactions were performed, according to manufacturer's instructions under the following thermocycler conditions: $50^{\circ} \mathrm{C}$ hold for 2 $\min , 95^{\circ} \mathrm{C}$ hold for $10 \mathrm{~min}$ followed by 40 cycles of $95^{\circ} \mathrm{C}$ for $15 \mathrm{~s}$, and $60^{\circ} \mathrm{C}$ for $1 \mathrm{~min}$. Template-negative controls were run on each PCR plate. A calibrator reverse-transcript sample was amplified in parallel on all plates in order to allow comparison of samples run at different times. The data were analyzed using Sequence Detection Software (PE Biosystems Japan), and the values were represented as mean \pm standard error (SE) of ratios (AQP/GAPDH mRNA amplicon) $\times 100 \%$.

\subsection{Immunohistochemistry}

Because the results of mRNA quantification revealed enhanced AQP8 expression in the remnant rectum of the subtotal colectomy group, immunohistochemistry for the remnant rectum of the subtotal colectomy group and for the rectum of the sham-operated group was carried out as described previously [13]. In brief, rat rectum samples were cut and fixed with methyl-Carnoy's fixative (60\% methanol, 30\% chroloform, 10\% acetic acid) overnight, dehydrated with ethanol, embedded in paraffin, and sectioned at $4 \mu \mathrm{m}$. The slide-sectioned tissues were deparaffinated with xylene and ethanol, hydrated in distilluted water, then blocked with normal goat serum (1:20 dilution) for $1 \mathrm{hr}$. After rinsing with PBS three times, the slides were incubated with anti-AQP8 antibody $(2.0 \mu \mathrm{g} /$ ml) (Alpha Diagnostic Intl. Inc., San Antonio, TX) for 1 hr at $37^{\circ} \mathrm{C}$ followed overnight at $4^{\circ} \mathrm{C}$. then rinsed with PBS three times, and incubated with goat anti-rabbit immunoglobulins conjugated to peroxidase labeled polymer (En Vision, DAKO, Kyoto, Japan), and colored by diaminobenzidine reaction. After being rinsed with distilluted water, the slides were counterstained with hematoxylin for observation.

\subsection{Statistical Analysis}

Mann-Whitney U-test was performed to compare the distributions between two groups. Kruskal-Wallis test was used to the equality of more than two group means and Bonferroni's correction to account for pairwise multiple comparisons. A two-tailed $\mathrm{P}$ value of $<0.05$ was considered to indicate statistical significance.

\section{RESULTS}

\subsection{Overall Animal Health}

The rats in both Group SB and Group Sham-SD lost body weight compared with normal animals (Group N). The body weight loss was more severe in Group SB compared to Group Sham-SD; however, it recovered at the end of the experiment (Figure 1). The subtotal co-

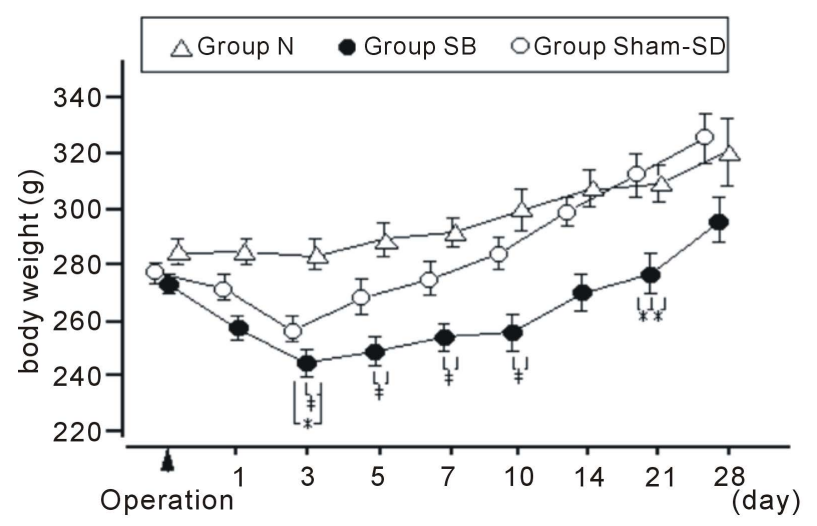

Figure 1. Body weight change in the short bowel model. The values are expressed as mean $\pm \mathrm{SE} .{ }^{*} \mathrm{p}<0.05$ and ${ }^{\ddagger} \mathrm{p}<0.01$ among the groups using Mann-Whitney U-test with corrected level of significance by Bonferroni method. 
lectomy model also showed marked body weight loss in Group TC compared to Group S-WKY (data not shown).

Watery stool was observed for 10 days postoperatively in both models of bowel resection (Group SB and Group TC) but was not observed in either model without bowel resection (Group Sham-SD and Group S-WKY).

\subsection{RNase Protection Assay}

In the short bowel model, the results of RNase protection assay showed no significant difference on AQP1, AQP3, AQP4 or AQP8 between the Group SB and Group ShamSD (Tables 1-4). In the Group SB, neither AQP1 nor AQP3 mRNA expression changed periodically during the experimental periods (Tables 1 and 2), however, both AQP4 and AQP8 mRNA expressions of the jejunum were elevated at day 7 followed by a gradual decrease during the experimental period (Table 3 and 4).

In the subtotal colectomy model, the AQP8 mRNA expression level was changed by bowel removal; AQP8 mRNA expression in the remnant rectum was obviously enhanced in Group TC at both day 21 and day 28, compared to Group S-WKY (Figure 2). No significant changes in AQP1, AQP3, and AQP4 mRNA expression were observed between Group TC and Group S-WKY (data not shown).

\subsection{Quantitative RT-PCR}

By significant quantitative RT-PCR analyses, no changes in AQP1, AQP3, and AQP4 mRNA expression were observed between Group SB and Group Sham-SD in the short bowel model, or between Group TC and Group SWKY in the subtotal colectomy model (data not shown), however, AQP8 mRNA expression showed statistically significant changes in both animal models.

In the short bowel model, AQP8 mRNA expression in the jejunum of Group SB, compared to that of Group Sham-SD, was enhanced at both day 7 (37.6\% $\pm 1.4 \%$, vs Sham-SD, p < 0.05) and day $14(18.5 \% \pm 2.4 \%$, vs Sham-SD, p < 0.05) (Figure 3). In Group SB, AQP8 mRNA expression in the jejunum was decreasing gradually, and was normalized by day 28 (37.6\% $\pm 1.4 \%$, 18.5\% $\pm 2.4 \%$, and $16.3 \% \pm 4.4 \%$, Kruskal-Wallis test, $\mathrm{p}<0.01$ ) (Figure 3). AQP8 mRNA of Group SB, compared to that of Group Sham-SD, was also enhanced in the proximal colon at day $7(20.7 \% \pm 4.8 \%$, vs Sham-SD, $\mathrm{p}<0.05)$, and was normalized by day $14(1.6 \% \pm 0.8 \%)$ (Figure 3$)$.

In the subtotal colectomy model, significant enhancement of AQP8 mRNA expression was observed in the remnant rectum of Group TC at both days 21 and 42 $(116.1 \% \pm 4.5 \%, \mathrm{p}<0.01$, and $143.3 \% \pm 7.4 \%, \mathrm{p}<0.01$, respectively) compared to Group S-WKY (Figure 4).

\subsection{Immunohistochemistry}

Immunohistochemistry using the anti-AQP8 antibody
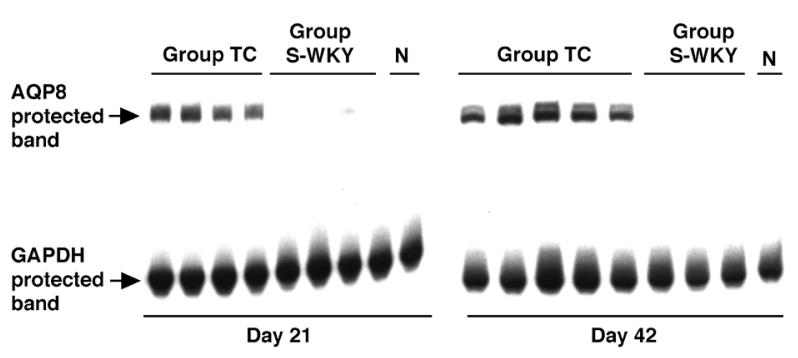

Figure 2. Expression of AQP8 mRNA in the remnant rectum in the subtotal colectomy model as examined by RNase protection assay. Group TC, subtotal colectomy group; Group S-WKY, sham-operated group, $\mathrm{N}$; normal rat.
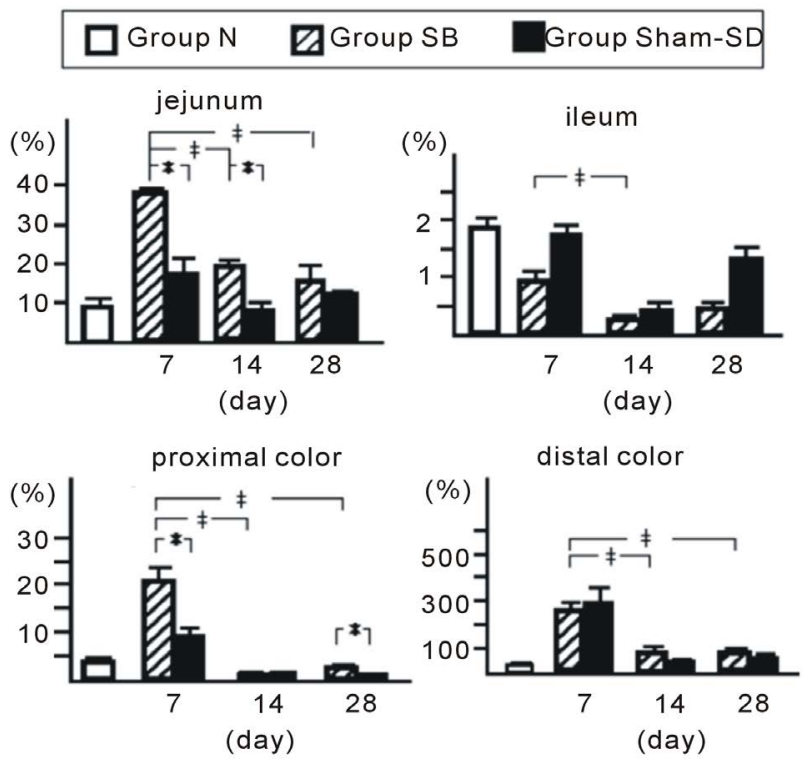

Figure 3. RT-PCR quantification of AQP8 mRNA expression in the jejunum, ileum, proximal colon, and distal colon in the short bowel model. The values are expressed as mean (SEM) of ratios (AQP/GAPDH mRNA amplicon) $\times 100 \%$. Kruskal-Wallis test is used to compare the distributions of three periods, and Mann-Whitney U-test with corrected level of significance by Bonferroni method used for pairwise multiple comparisons. ${ }^{*}$ and ${ }^{\ddagger}$ represent $\mathrm{p}<0.05$ and $\mathrm{p}<0.01$, respectively.

demonstrated prominent AQP8 protein expression in Group TC. AQP8 was expressed mainly in the cytoplasm of columunar epithelial cells of the remnant rectum (Figures 5(a) and (b)). AQP8 staining was negligible in the rectums of sham-operated rats (Figures 5(c) and 5(d)).

\section{DISCUSSION}

Since the discovery of AQP1, 13 mammalian AQPs have been recognized, and clinical or physiological importance of AQPs has been shown or suggested; AQP0 mutations have been identified in some forms of congenital cataracts [14]. AQP1-null individuals have shown limited 
Table 1. Quantification of AQP1 mRNA expression in the short bowel model by RNase protection assay.

\begin{tabular}{cccccccc}
\hline & Group N & \multicolumn{3}{c}{ Group SB } & \multicolumn{3}{c}{ Group Sham-SD } \\
\hline & & Day 7 & Day 14 & Day 28 & Day 7 & Day 14 & Day 28 \\
Jejunum & $3.9 \pm 1.8$ & $10.6 \pm 4.2$ & $23.8 \pm 7.7$ & $6.3 \pm 1.1$ & $4.3 \pm 1.7$ & $28.7 \pm 12.0$ & $6.7 \pm 1.3$ \\
Ileum & $16.8 \pm 4.1$ & $3.0 \pm 1.6^{\dagger}$ & $8.9 \pm 2.2$ & $14.1 \pm 3.1$ & $16.7 \pm 9.4$ & $23.4 \pm 2.3$ & $33.1 \pm 4.1$ \\
Proximal colon & $11.3 \pm 2.4$ & $4.9 \pm 1.8$ & $6.9 \pm 1.6$ & $10.7 \pm 2.6$ & $5.4 \pm 1.8$ & $6.2 \pm 0.3$ & $11.3 \pm 2.4$ \\
Distal colon & $7.7 \pm 0.6$ & $10.2 \pm 2.3$ & $15.8 \pm 4.3$ & $13.4 \pm 0.9$ & $7.0 \pm 1.9$ & $22.0 \pm 1.7$ & $13.2 \pm 1.0$ \\
\hline
\end{tabular}

The values are expressed as mean \pm SE of ratios (AQP/GAPDH mRNA densitometric unit) $\times 100 \%$. In the Group SB, ${ }^{\dagger} \mathrm{p}<0.05$ between Day 7 and Day 28 , by Mann-Whitney U-test and Kruskal-Wallis method.

Table 2. Quantification of AQP3 mRNA expression in the short bowel model by RNase protection assay.

\begin{tabular}{cccccccc}
\hline & Group N & \multicolumn{3}{c}{ Group SB } & \multicolumn{3}{c}{ Group Sham-SD } \\
\hline & & Day 7 & Day 14 & Day 28 & Day 7 & Day 14 & Day 28 \\
Jejunum & $1.9 \pm 0.3$ & $4.7 \pm 1.3^{\dagger}$ & $8.1 \pm 2.7$ & $0.9 \pm 0.7$ & $3.1 \pm 0.6$ & $6.2 \pm 0.8$ & $0.6 \pm 0.4$ \\
Ileum & $15.0 \pm 0.6$ & $5.5 \pm 1.3$ & $6.2 \pm 0.6$ & $2.0 \pm 0.9$ & $3.5 \pm 3.1$ & $6.0 \pm 1.9$ & $4.3 \pm 1.9$ \\
Proximal colon & $5.4 \pm 1.5$ & $1.4 \pm 0.6$ & $4.3 \pm 1.3$ & $1.9 \pm 0.6$ & $5.7 \pm 3.0$ & $5.9 \pm 0.7$ & $4.7 \pm 1.5$ \\
Distal colon & $13.0 \pm 1.3$ & $5.4 \pm 2.2^{\dagger}$ & $7.7 \pm 2.2^{\#}$ & $26.0 \pm 2.9$ & $4.7 \pm 4.1$ & $8.7 \pm 1.5$ & $26.1 \pm 2.8$ \\
\hline
\end{tabular}

The values are expressed as mean \pm SE of ratios (AQP/GAPDH mRNA densitometric unit) $\times 100 \%$. In the Group SB, ${ }^{\dagger} \mathrm{p}<0.05$ between Day 7 and Day 28 , and $\#$ p $<0.05$ between Day 14 and Day 28, by Mann-Whitney U-test and Kruskal-Wallis method.

Table 3. Quantification of AQP4 mRNA expression in the short bowel model by RNase protection assay.

\begin{tabular}{cccccccc}
\hline & Group N & \multicolumn{3}{c}{ Group SB } & \multicolumn{3}{c}{ Group Sham-SD } \\
\hline & & Day 7 & Day 14 & Day 28 & Day 7 & Day 14 & Day 28 \\
Jejunum & $2.6 \pm 0.7$ & $3.9 \pm 1.0^{\dagger}$ & $2.9 \pm 0.3^{\#}$ & $0.5 \pm 0.3$ & $1.3 \pm 1.3$ & $3.4 \pm 0.4$ & $0.6 \pm 0.6$ \\
Ileum & $11.2 \pm 2.6$ & $5.3 \pm 0.2$ & $1.8 \pm 0.7$ & $6.7 \pm 1.6$ & $8.6 \pm 0.9$ & $3.6 \pm 0.5$ & $12.0 \pm 2.0$ \\
Proximal colon & ND & ND & ND & ND & ND & ND & ND \\
Distal colon & ND & ND & ND & ND & ND & ND & ND \\
\hline
\end{tabular}

The values are expressed as mean $\pm \mathrm{SE}$ of ratios (AQP/GAPDH mRNA densitometric unit) $\times 100 \%$. In the Group SB, ${ }^{\dagger} \mathrm{p}<0.05$ between Day 7 and Day 28 , and \# $<0.05$ between Day 14 and Day 28, by Mann-Whitney U-test and Kruskal-Wallis method. ND: not detected.

Table 4. Quantification of AQP8 mRNA expression in the short bowel model by RNase protection assay.

\begin{tabular}{cccccccc}
\hline & Group N & \multicolumn{3}{c}{ Group SB } & \multicolumn{3}{c}{ Group Sham-SD } \\
\hline & & Day 7 & Day 14 & Day 28 & Day 7 & Day 14 & Day 28 \\
Jejunum & $5.8 \pm 0.6$ & $11.1 \pm 2.2^{\dagger}$ & $8.4 \pm 1.1^{\#}$ & $2.9 \pm 0.6$ & $5.3 \pm 1.4$ & $4.8 \pm 0.1$ & $4.5 \pm 1.4$ \\
Ileum & ND & ND & ND & ND & ND & ND & ND \\
Proximal colon & $12.7 \pm 3.1$ & $7.5 \pm 1.6$ & $4.0 \pm 0.9$ & $2.6 \pm 0.3$ & $9.1 \pm 1.9$ & $4.1 \pm 1.0$ & $2.5 \pm 0.4$ \\
Distal colon & $35.2 \pm 4.8$ & $33.5 \pm 6.9$ & $35.6 \pm 4.7$ & $29.0 \pm 4.7$ & $8.6 \pm 1.9$ & $22.9 \pm 5.2$ & $18.0 \pm 5.1$ \\
\hline
\end{tabular}

The values are expressed as mean \pm SE of ratios (AQP/GAPDH mRNA densitometric unit) $\times 100 \%$.In the Group SB, ${ }^{\dagger}$ p $<0.05$ between Day 7 and Day 28 , and \# $<0.05$ between Day 14 and Day 28, By Mann-Whitney U-test and Kruskal-Wallis method. ND: not detected.

ability to maximally concentrate urine under water deprived conditions [15], and have shown decreased pulmonary vascular permeability [16]. AQP2 mutation causes clinical congenital diabetes insipidus [17], and AQP3 and AQP4 seem to play a role in urinary concentration in knockout mice studies [18,19]. Abnormalities of AQP5 distribution have shown to be related to Sjogren's syndrome [20]. Although several experimental models using transgenic mice lacking AQPs have been examined previously [21], the functions of AQPs in digestive organs remain unclear.

Previously we have shown the expression, distribution, and localization of AQPs in rat digestive organs including the GI tract: Generalized expressions of AQP1 and AQP3 mRNA are expressed widely along the gastrointestinal tract, AQP4 mRNA is expressed selectively in the lower portion of stomach and the small intestine, and AQP8 mRNA is expressed more selectively in the jejunum and colon [12,13]. We have also cloned AQP10 from the human jejunum. AQP10 was selectively expressed in upper sites of the small intestine such as the duodenum and jejunum in humans [11].

In the present study, we performed a quantitative investigation using both RNase protection assay and RT-PCR 


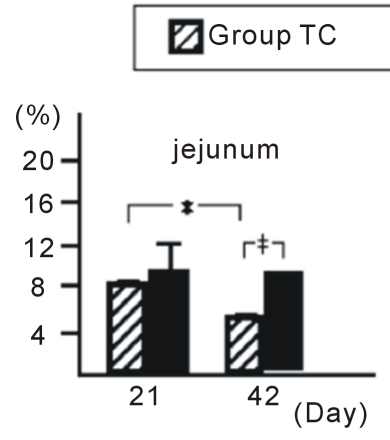

Group S-WKY
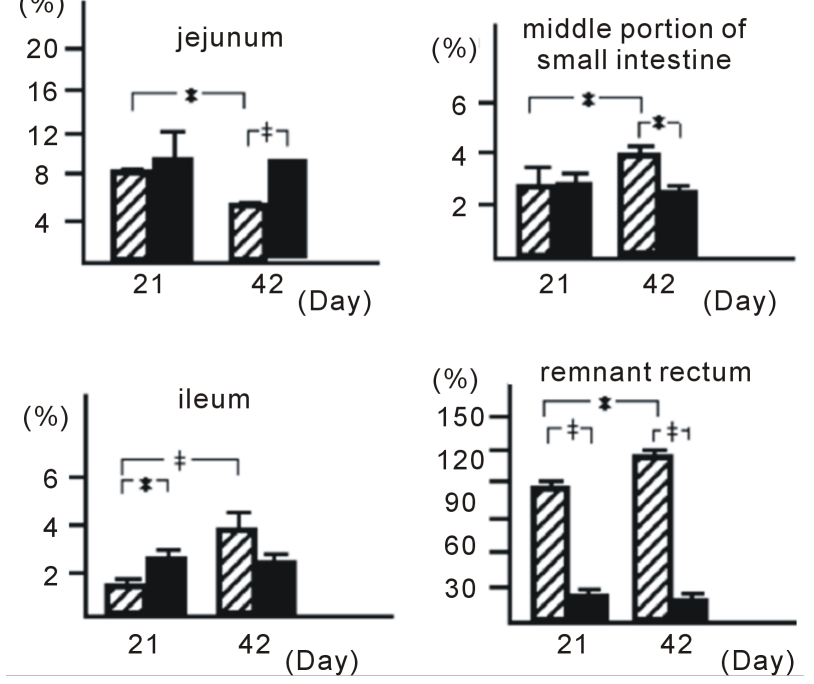

Figure 4. RT-PCR quantification of AQP8 mRNA expression in the jejunum, middle portion of small intestine, ileum, and remnant rectum in the subtotal colectomy model. The values are expressed as mean (SEM) of ratios (AQP/GAPDH mRNA amplicon) $\times 100 \% .{ }^{*} \mathrm{p}<0.05$ and ${ }^{\ddagger} \mathrm{p}<0.01$ between the two groups by Mann-Whitney U-test.
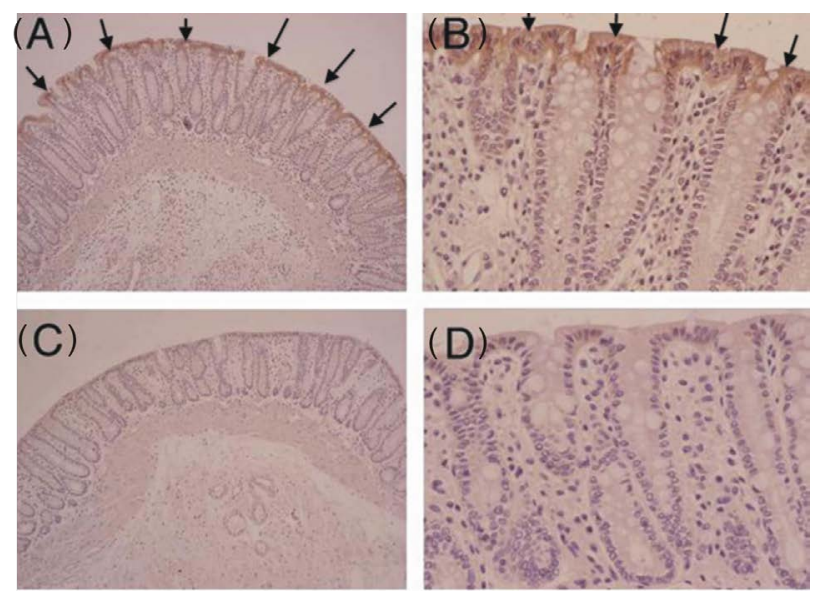

Figure 5. Immunohistochemistry of AQP8 protein expression in the rectum of the subtotal colectomy model. (A) remnant rectum of Group TC at day 21. (B) remnant rectum of Group TC at day 42. (C) rectum of Group S-WKY at day 21. (D) rectum of Group S-WKY at day 42. (magnification (A) and (C): 100×; (B) and (D): 400×). Enhanced AQP8 protein expression (arrow) was detected in the apical membrane of remnant rectum of Group TCat both day 21 and day 42.

to elucidate the changes of AQP family gene expression of mRNA in the rat gastrointestinal tract after bowel removal. One of our results showed increased AQP8 mRNA expression in the jejunum of the short bowel model at an early stage of the postoperative course, suggesting that
AQP8, in the jejunum, plays a role of water movement between jejunal mucosa and lumen in order to dilute/ absorb the intestinal contents under short bowel condition. Another result, prominent enhancement of AQP8 mRNA expression in the remnant rectum of the subtotal colectomized rats, suggests that AQP8, in the rectum, is useful for water movement in the short length of the colon.

On the other hand, no significant changes of AQP1 and AQP4 mRNA expression were observed in the GI tract in either experimental model (data not shown). Moreover, AQP3 has been suggested to function in the small and the large intestine by previous studies [22,23]. However, significant change of AQP3 mRNA expression was not demonstrated in the present models.

We also used immunohistochemistry to confirm the enhancement of AQP8 protein in the rectum of the subtotal colectomized rats being in accordance with the enhancement of AQP8 mRNA expression. Our results showed prominent AQP8 protein on the columunar epithelial cells of the rectum of the subtotal colectomized rats, while the staining was negligible in the sham-operated rats. The cytoplasmic localization of AQP8 in the present study was different from that described in our previous report, in which AQP8 was localized on the apical membrane of the columunar jejunum and colonic epithelial cells $[12,13]$. It is not clear about this discrepancy of AQP8 localization. Further studies including an AQP8 knockout model are expected to elucidate AQP8 function and localization.

Physiological roles of water channels in water transportation through the epithelial cell layer in the GI tract are still unclear. However, our results suggest an important role of AQP8 in the maintenance of intestinal fluid balance, and for adaptation to postoperative conditions.

\section{ACKNOWLEDGEMENTS}

We thank Mr. Kan Yoshida for his technical assistance. This work was supported in part by Grants-in-Aid for Science Research from the Ministry of Education, Science, Sports and Culture, Japan (08671342 \& 09470237).

\section{REFERENCES}

[1] King, L.S. and Agre, P. (1996) Pathophysiology of the aquaporin water channels. Annual Review of Physiology, 58, 619-648.

http://dx.doi.org/10.1146/annurev.ph.58.030196.003155

[2] Preston, G.M., Carroll, T.P., Guggino, W.B. and Agre, P. (1992) Appearance of water channels in Xenopus oocytes expressing red cell CHIP28 protein. Science, 256, 385387. http://dx.doi.org/10.1126/science.256.5055.385

[3] Fushimi, K., Uchida, S., Hara, Y., Hirata, Y., Marumo, F. and Sasaki, S. (1993) Cloning and expression of apical 
membrane water channel of rat kidney collecting tubule. Nature, 361, 549-552.

http://dx.doi.org/10.1038/361549a0

[4] Ishibashi, K., Sasaki, S., Fushimi, K., Uchida, S., Kuwahara, M., Saito, H., Furukawa, T., Nakajima, K., Yamaguchi, Y., Gojobori, T. and Marumo, F. (1994) Molecular cloning and expression of a member of the aquaporin family with permeability to glycerol and urea in addition to water expressed at the basolateral membrane of kidney collecting duct cells. Proceedings of the National Academy of Sciences USA, 91, 6269-6273.

[5] Jung, J.S., Bhat, R.V., Preston, G.M., Guggino, W.B., Baraban, J.M. and Agre, P. (1994) Molecular characterization of an aquaporin cDNA from brain: Candidate osmoreceptor and regulator of water balance. Proceedings of the National Academy of Sciences USA, 91, 1305213056.

[6] Raina, S., Preston, G.M., Guggino, W.B. and Agre, P. (1995) Molecular cloning and characterization of an aquaporin cDNA from salivary, lacrimal, and respiratory tissues. Journal of Biological Chemistry, 270, 1908-1912. http://dx.doi.org/10.1074/jbc.270.4.1908

[7] Ma, T., Yang, B., Kuo, W.L. and Verkman, A.S. (1996) cDNA cloning and gene structure of a novel water channel expressed exclusively in human kidney: Evidence for a gene cluster of aquaporins at chromosome locus 12q13. Genomicus, 35, 543-550.

http://dx.doi.org/10.1006/geno.1996.0396

[8] Ishibashi, K., Kuwahara, M., Gu, Y., Kageyama, Y., Tohsaka, A., Suzuki, F., Marumo, F. and Sasaki, S. (1997) Cloning and functional expression of a new water channel abundantly expressed in the testis permeable to water, glycerol, and urea. Journal of Biological Chemistry, 272, 20782-20786. http://dx.doi.org/10.1074/jbc.272.33.20782

[9] Koyama, Y., Yamamoto, T., Kondo, D., Funaki, H., Yaoita, E., Kawasaki, K., Sato, N., Hatakeyama, K. and Kihara, I. (1997) Molecular cloning of a new aquaporin from rat pancreas and liver. Journal of Biological Chemistry, 272, 30329-30333.

http://dx.doi.org/10.1074/jbc.272.48.30329

[10] Ishibashi, K., Kuwahara, M., Gu, Y., Tanaka, Y., Marumo, F. and Sasaki, S. (1998) Cloning and functional expression of a new aquaporin (AQP9) abundantly expressed in the peripheral leukocytes permeable to water and urea, but not to glycerol. Biochemical and Biophysical Research Communications, 244, 268-274.

http://dx.doi.org/10.1006/bbrc.1998.8252

[11] Hatakeyama, S., Yoshida, Y., Tani, T., Koyama, Y., Nihei, K., Ohshiro, K., Kamiie, J.I., Yaoita, E., Suda, T., Hatakeyama, K. and Yamamoto, T. (2001) Cloning of a new aquaporin (aqp10) abundantly expressed in duodenum and jejunum. Biochemical and Biophysical Research Communications, 287, 814-819.

http://dx.doi.org/10.1006/bbrc.2001.5661

[12] Koyama, Y., Yamamoto, T., Tani, T., Nihei, K., Kondo, D., Funaki, H., Yaoita, E., Kawasaki, K., Sato, N., Hatakeyama, K. and Kihara, I. (1999) Expression and localization of aquaporins in rat gastrointestinal tract. American Journal of Physiology, 276, C621-C627.
[13] Tani, T., Koyama, Y., Nihei, K., Hatakeyama, S., Ohshiro, K., Yoshida, Y., Yaoita, E., Sakai, Y., Hatakeyama, K. and Yamamoto, T. (2001) Immunolocalization of aquaporin-8 in rat digestive organs and testis. Archives of Histology and Cytololgy, 64, 159-168. http://dx.doi.org/10.1679/aohc.64.159

[14] Berry, V., Francis, P., Kaushal, S., Moore, A. and Bhattacharya, S. (2000) Missense mutations in MIP underlie autosomal dominant 'polymorphic' and lamellar cataracts linked to 12q. Nauret Genetics, 25, 15-17. http://dx.doi.org/10.1038/75538

[15] King, L.S., Choi, M., Fernandez, P.C., Cartron, J.P. and Agre, P. (2001) Defective urinary-concentrating ability due to a complete deficiency of aquaporin-1. New England Journal of Medicine, 345, 175-179. http://dx.doi.org/10.1056/NEJM200107193450304

[16] King, L.S., Nielsen, S., Agre, P. and Brown, R.H. (2002) Decreased pulmonary vascular permeability in aquaporin1-null humans. Proceedings of the National Academy of Sciences USA, 99, 1059-1063.

[17] Deen, P.M., Verdijk, M.A., Knoers, N.V., Wieringa, B., Monnens, L.A., van Os, C.H. and van Oost, B.A. (1994) Requirement of human renal water channel aquaporin-2 for vasopressin-dependent concentration of urine. Science, 264, 92-95. http://dx.doi.org/10.1126/science.8140421

[18] Ma, T., Yang, B., Gillespie, A., Carlson, E.J., Epstein, C.J. and Verkman, A.S. (1997) Generation and phenotype of a transgenic knockout mouse lacking the mercurial-insensitive water channel aquaporin-4. Journal of Clinical Investigation, 100, 957-962.

http://dx.doi.org/10.1172/JCI231

[19] Ma, T., Song, Y., Yang, B., Gillespie, A., Carlson, E.J., Epstein, C.J. and Verkman, A.S. (2000) Nephrogenic diabetes insipidus in mice lacking aquaporin-3 water channels. Proceedings of the National Academy of Sciences USA, 97, 4386-4391.

[20] Steinfeld, S., Cogan, E., King, L.S., Agre, P., Kiss, R. and Delporte, C. (2001) Abnormal distribution of aquaporin-5 water channel protein in salivary glands from Sjögren's syndrome patients. Laboratory Investigation, 81, 143148. http://dx.doi.org/10.1038/labinvest.3780221

[21] Verkman, A.S., Yang, B., Song, Y., Manley, G.T. and Ma, T. (2000) Role of water channels in fluid transport studied by phenotype analysis of aquaporin knockout mice. Experimental Physiology, 85, 233S-241S. http://dx.doi.org/10.1111/j.1469-445X.2000.tb00028.x

[22] Silberstein, C., Kierbel, A., Amodeo, G., Zotta, E., Bigi, F., Berkowski, D. and Ibarra, C. (1999) Functional characterization and localization of AQP3 in the human colon. Brazilian Journal of Medical and Biological Research, 32, 1303-1313. http://dx.doi.org/10.1590/S0100-879X1999001000018

[23] Purdy, M.J., Cima, R.R., Doble, M.A., Klein, M.A., Zinner, M.J. and Soybel, D.I. (1999) Selective decrease in levels of mRNA encoding a water channel (AQP3) in ileal mucosa after ileostomy in the rat. Journal of Gastrointestinal Surgery, 3, 54-60. http://dx.doi.org/10.1016/S1091-255X(99)80009-2 